\title{
Targeting $\mathrm{NAD}^{+}$Metabolism in the Human Malaria Parasite Plasmodium falciparum
}

\author{
Jessica K. O'Hara', Lewis J. Kerwin ${ }^{2}$, Simon A. Cobbold ${ }^{1}$, Jonathan Tai ${ }^{3}$, Thomas A. Bedell' \\ Paul J. Reider ${ }^{2}$, Manuel Llinás ${ }^{1 * *}$
}

1 Department of Molecular Biology and Lewis-Sigler Institute for Integrative Genomics, Princeton University, Princeton, New Jersey, United States of America, 2 Department of Chemistry, Princeton University, Princeton, New Jersey, United States of America, 3 Department of Biochemistry, University of Illinois at UrbanaChampaign, Urbana, Illinois, United States of America

\begin{abstract}
Nicotinamide adenine dinucleotide $\left(\mathrm{NAD}^{+}\right)$is an essential metabolite utilized as a redox cofactor and enzyme substrate in numerous cellular processes. Elevated $\mathrm{NAD}^{+}$levels have been observed in red blood cells infected with the malaria parasite Plasmodium falciparum, but little is known regarding how the parasite generates $\mathrm{NAD}^{+}$. Here, we employed a mass spectrometry-based metabolomic approach to confirm that $P$. falciparum lacks the ability to synthesize $N^{+} D^{+}$de novo and is reliant on the uptake of exogenous niacin. We characterized several enzymes in the NAD ${ }^{+}$pathway and demonstrate cytoplasmic localization for all except the parasite nicotinamidase, which concentrates in the nucleus. One of these enzymes, the $P$. falciparum nicotinate mononucleotide adenylyltransferase (PfNMNAT), is essential for NAD ${ }^{+}$metabolism and is highly diverged from the human homolog, but genetically similar to bacterial NMNATs. Our results demonstrate the enzymatic activity of PfNMNAT in vitro and demonstrate its ability to genetically complement the closely related Escherichia coli NMNAT. Due to the similarity of PfNMNAT to the bacterial enzyme, we tested a panel of previously identified bacterial NMNAT inhibitors and synthesized and screened twenty new derivatives, which demonstrate a range of potency against live parasite culture. These results highlight the importance of the parasite $N^{+} D^{+}$metabolic pathway and provide both novel therapeutic targets and promising lead antimalarial compounds.
\end{abstract}

Citation: O'Hara JK, Kerwin LJ, Cobbold SA, Tai J, Bedell TA, et al. (2014) Targeting NAD ${ }^{+}$Metabolism in the Human Malaria Parasite Plasmodium falciparum. PLoS ONE 9(4): e94061. doi:10.1371/journal.pone.0094061

Editor: Frank Voncken, University of Hull, United Kingdom

Received November 25, 2013; Accepted March 11, 2014; Published April 18, 2014

Copyright: (c) 2014 O'Hara et al. This is an open-access article distributed under the terms of the Creative Commons Attribution License, which permits unrestricted use, distribution, and reproduction in any medium, provided the original author and source are credited.

Funding: This work was funded through generous support from the Burrough's Wellcome fund (Investigators in Pathogenesis of Infectious Disease Award for Research), an NIH Director's New Innovator Award (1DP2OD001315-01), and with support from the Center for Quantitative Biology (P50 GM071508). The funders had no role in study design, data collection and analysis, decision to publish, or preparation of the manuscript.

Competing Interests: The authors have declared that no competing interests exist.

*E-mail: manuel@psu.edu

a Current address: Department of Biochemistry and Molecular Biology, Penn State University, University Park, Pennsylvania, United States of America

\section{Introduction}

Malaria remains one of the most devastating and prevalent infectious diseases worldwide, with 350 to 500 million annual cases, imposing a heavy burden on the healthcare and economic development of afflicted countries [1,2]. The Apicomplexan parasite Plasmodium falciparum is responsible for the most severe form of malaria killing 650,000 individuals in 2011, with $86 \%$ of deaths occurring in children under the age of five [3]. The recent rise in drug resistant parasite strains has increased the burden of malaria and drawn attention to the need for the identification of novel drug targets and new antimalarial therapeutics. Many of the clinical symptoms of malaria are tied to the metabolic stresses placed on the host when the parasite infects and develops within the red blood cell. As the parasite rapidly grows and divides during its 48 hour asexual life cycle it is greatly dependent on glycolysis for energy production. Plasmodium-infected erythrocytes can consume glucose at approximately one hundred times the rate of uninfected erythrocytes $[4,5]$. This primary reliance on anaerobic respiration is coincidental with some of the most distinguishable clinical symptoms associated with malaria, such as hypoglycemia and lactic acidosis. Therefore, increased characterization of the poorly understood metabolism of $P$. falciparum is important to understand many of the host-parasite interactions that underlie the clinical symptoms of malaria and for identifying both novel pathways and specific enzymes to target therapeutically.

Studies in four different Plasmodium species have previously reported that $\mathrm{NAD}^{+}$levels are high during the asexual blood stage of development, with infected erythrocytes exhibiting 5 to 10 -fold higher concentrations compared to uninfected red blood cells [69]. $\mathrm{NAD}^{+}$and its phosphorylated $\left(\mathrm{NADP}^{+}\right)$and reduced forms (NADH and NADPH) are essential to the central metabolism of all organisms and are well understood for their role as important redox cofactors [10]. In recent years, however, $\mathrm{NAD}^{+}$has gained recognition for its diverse role as an enzyme substrate in a number of essential cellular processes including epigenetic regulation, calcium signaling, and DNA repair $[11,12]$. The $P$. falciparum genome appears to encode significantly fewer $\mathrm{NAD}^{+}$utilizing enzymes than other organisms, containing only two putative sirtuin proteins (Sir2) and no homologs of poly(ADP-ribose) polymerase [13]. Sir2 proteins catalyze the deacetylation of proteins, most notably histones, in a $\mathrm{NAD}^{+}$-dependent manner [14]. In P. falciparum the two Sir2 proteins (PF13_0152, PfSIR2A and PF14_0489, PfSIR2B) are involved in telomere maintenance 
and genetic regulation of the subtelomeric var gene family, which encodes the $P$. falciparum erythrocyte membrane protein 1 (PfEMPl) surface protein, an important factor in parasite cytoadherence and virulence [15-18]. Due to the catabolic $\mathrm{NAD}^{+}$requirement by the $\mathrm{Sir} 2 \mathrm{~s}$ [14], and the requirement of $\mathrm{NAD}^{+}$as a cofactor for many other $\mathrm{NAD}^{+}$dependent enzymes, it is likely that regulation of the $\mathrm{NAD}^{+}$metabolic pathway provides a link between metabolism and a variety of important cellular processes in the Plasmodium parasite.

$\mathrm{NAD}^{+}$can be synthesized in most organisms through both salvage and de novo pathways. In the human red blood cell, $\mathrm{NAD}^{+}$ synthesis is limited to a $\mathrm{NAD}^{+}$salvage pathway that utilizes either exogenously acquired nicotinic acid $(\mathrm{Na})$ or nicotinamide (Nam), which are collectively known as niacin or vitamin $\mathrm{B}_{3}$ [19]. $\mathrm{Na}$ is converted to $\mathrm{NAD}^{+}$through the Preiss-Handler pathway in three steps - $\mathrm{Na}$ is first converted into nicotinate mononucleotide $(\mathrm{NaMN})$ via the nicotinic acid phosphoribosyltransferase (NAPRT), then to nicotinate adenine dinucleotide (NaAD) via the nicotinamide mononucleotide adenylyltransferase (NMNAT) and finally to $\mathrm{NAD}^{+}$via the glutamine-dependent $\mathrm{NAD}^{+}$ synthetase (NADSYN) [20,21] - while Nam can be converted to $\mathrm{NAD}^{+}$in a two-step pathway found in higher eukaryotic organisms involving nicotinamide riboside kinase (NRK) and NMNAT (Figure 1A) [22]. In the de novo synthesis pathway, prokaryotes can utilize aspartate to feed into the synthesis of $\mathrm{NAD}^{+}$, whereas eukaryotes rely on intermediates generated from the breakdown of tryptophan [23]. Both pathways yield NaMN, which ultimately enters into the final two steps of the salvage pathway. The $P$. falciparum genome is currently predicted to only encode the enzymes necessary for a functional $\mathrm{NAD}^{+}$salvage pathway (NAPRT: PFF1410c, NMNAT: PF13_0159 and NADSYN: PFI1310w) (Figure 1A) and does not appear to possess the enzymes for de novo synthesis from either aspartate or tryptophan $[13,24]$. While both the host and parasite possess the $\mathrm{NAD}^{+}$ salvage pathway, there is a significant divergence between the two pathways due to the presence of a nicotinamidase enzyme (PFC0910w) in the P. falciparum genome that is able to convert Nam to Na (Figure 1A).

Our current study explores the broader significance of $\mathrm{NAD}^{+}$ metabolism in P. falciparum throughout blood-stage development as a target for antimalarial intervention. Using a combination of protein biochemistry, genetics, and mass spectrometry-based metabolomics approaches, we have characterized the $\mathrm{NAD}^{+}$ metabolic pathway of the parasite confirming the absence of a $d e$ novo synthetic route. All four enzymes of the parasite salvage pathway have been localized, revealing a cytoplasmic localization for all enzymes but nicotinamidase, which localizes to the nucleus. We enzymatically characterized the parasite nicotinate mononucleotide adenylyltransferase (PfNMNAT) in vitro using purified recombinant protein and we demonstrate its ability to complement the Escherichia coli homolog in vivo. Following up on previous predictions that PfNMNAT is essential to $P$. falciparum development [25], we synthesized and screened derivatives of previously identified inhibitors of bacterial NMNATs [26] against purified PfNMNAT and live parasite culture. This work validates the parasite $\mathrm{NAD}^{+}$metabolic pathway as a novel drug target and identifies viable lead compounds for further development as antimalarial drugs.

\section{Results}

\section{Characterization of parasite $\mathrm{NAD}^{+}$metabolism}

Genomic reconstruction of Plasmodium falciparum has identified a number of enzymes involved in $\mathrm{NAD}^{+}$metabolism [24], suggest- ing that the parasite uses a canonical Preiss-Handler salvage pathway, but lacks the enzymes required for de novo synthesis (Figure 1A). The parasite genome encodes for a nicotinamidase (PFC0910w, EG 3.5.1.19), nicotinate phosphoribosyltransferase (PFF1410c, EG 2.4.2.11), nicotinate mononucleotide adenylyltransferase (PF13_0159, EC 2.7.7.18), and $\mathrm{NAD}^{+}$synthase (PFI1310w, EC 6.3.5.1). To identify the subcellular localization of $\mathrm{NAD}^{+}$metabolism, P. falciparum 3D7 strains were generated expressing full-length GFP-tagged episomal copies of each enzyme and then analyzed by live fluorescence microscopy. We found that the $\mathrm{NAD}^{+}$pathway enzymes are primarily localized in the cytoplasm except for the parasite nicotinamidase, which localizes to the cytoplasm but concentrates mainly in the nucleus (Figure 1B and Figure $\mathrm{S} 1$ in File $\mathrm{S} 1$ ).

In order to confirm the predicted architecture of the $P$. falciparum $\mathrm{NAD}^{+}$salvage pathway, we grew infected red blood cells (iRBCs) in media supplemented with uniformly labeled ${ }^{13} \mathrm{C}$-U-glucose to perform stable isotopic labeling studies coupled with liquid chromatography-tandem mass spectrometry (LC-MS/MS) detection. Under these conditions ${ }^{13} \mathrm{C}$-U-labeled glucose is converted into ${ }^{13} \mathrm{C}$-U-labeled phosphoribosyl pyrophosphate (PRPP) via the pentose phosphate pathway, which is then incorporated into the nucleotide components of $\mathrm{NAD}^{+}$; nicotinate/nicotinamide mononucleotide $(\mathrm{N}(\mathrm{a}) \mathrm{MN})$ and adenosine (as shown in Figure $\mathrm{S} 2$ in File $\mathrm{S} 1$ ). Using this approach, we are able to monitor all of the $\mathrm{NAD}^{+}$ salvage pathway intermediates and observe the labeling of $\mathrm{NAD}^{+}$ throughout the stages of the intraerythrocytic developmental cycle (IDC). The incorporation of $5+$ and $10+$ label into newly synthesized $\mathrm{NAD}^{+}$occurs linearly in both infected red blood cells (iRBCs) and uninfected red blood cells ( $\mathrm{uRBCs}$ ), but is faster in the infected cell $\left(\mathrm{d}\left[\%{ }^{13} \mathrm{C}-\mathrm{NAD}+\right] / \mathrm{dt}=0.02 \pm 0.00\right)$ than the uninfected cell $\left(\mathrm{d}\left[\%{ }^{13} \mathrm{C}-\mathrm{NAD}+\right] / \mathrm{dt}=0.01 \pm 0.01\right)$ (Figure $\left.2 \mathrm{~A}\right)$. In addition to the steady synthesis of $\mathrm{NAD}^{+}$, turnover of unlabeled $\mathrm{NAD}^{+}$was also observed over the 48 hour time course in both uninfected and infected cells by the steady decrease in pool of unlabeled $\mathrm{NAD}^{+}$ (Figure 2A). The predominant form of $\mathrm{NAD}^{+}$generated in iRBCs contains newly synthesized nicotinic acid mononucleotide (NaMN) and unlabeled ATP (shown in Figure S3 in File S1). This is expected, as all NaMN made by the parasite will be incorporated into $\mathrm{NAD}^{+}$, while ATP is utilized in a number of additional cellular pathways.

A comparison of steady state levels of metabolic intermediates demonstrates that uninfected red blood cells preferentially generate $\mathrm{NAD}^{+}$via nicotinamide mononucleotide $(\mathrm{NMN})$, while the parasite produces $\mathrm{NAD}^{+}$via the canonical Preiss-Handler twostep process, yielding both the labeled intermediates $\mathrm{NaMN}$ and nicotinate adenine dinucleotide $(\mathrm{NaAD})$. This architecture was confirmed by the large fold increases observed in labeled NaMN and NaAD produced by the parasite compared to uRBCs, while labeled NMNs levels generated by the red blood cell were relatively unchanged in the presence of the parasite (Figure 2B). Therefore, the large increase in $\mathrm{NAD}^{+}$concentration previously observed during Plasmodium infection is due to parasite-derived metabolism. The production of ${ }^{13} \mathrm{C}$-labeled $\mathrm{NAD}^{+}$remains steady throughout the IDC in iRBCs with a total intracellular concentration of $880 \pm 100 \mu \mathrm{M}$ during the schizont stage, almost twenty times higher than the concentration measured in uninfected controls $(44 \pm 6 \mu \mathrm{M})$ (Table 1). While $\mathrm{NAD}^{+}$levels are greatly increased in the infected red blood cell, $\mathrm{NADP}^{+}$concentrations are only slightly increased from $35 \pm 1 \mu \mathrm{M}$ to $62 \pm 12 \mu \mathrm{M}$ upon infection (Table 1). Despite the amount of labeled $\mathrm{NAD}^{+}$ increasing by almost $100 \%$ in the $\mathrm{iRBC}$ during development (Figure 2A), only a small fraction of this pool is phosphorylated to 


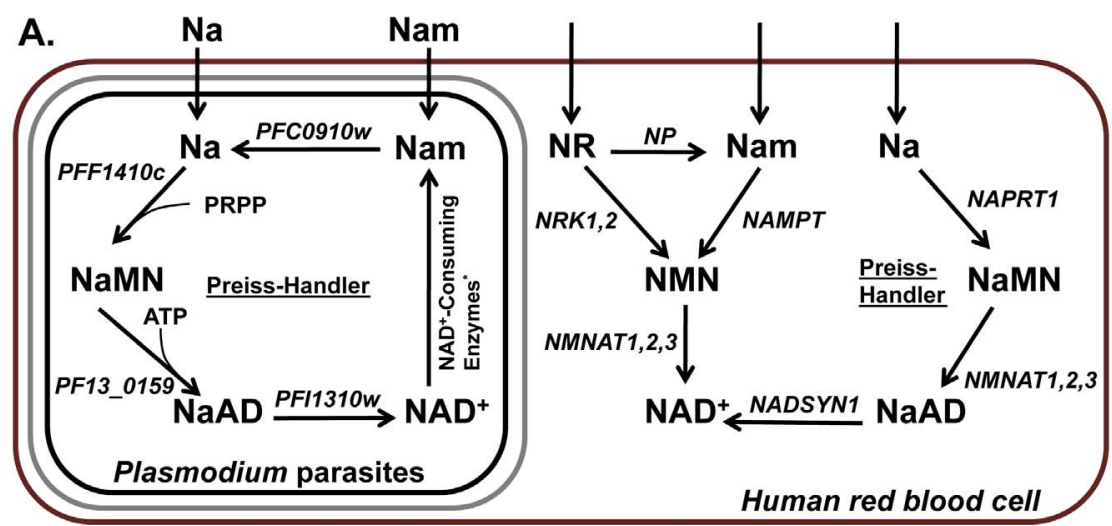

B.

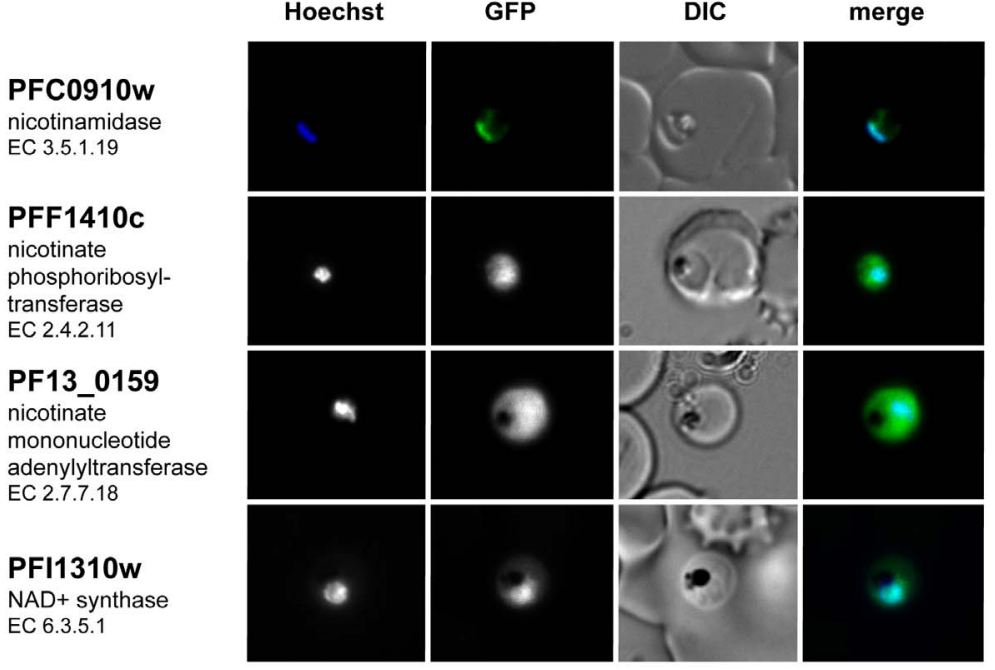

Figure 1. Plasmodium falciparum NAD $^{+}$metabolism. A. Overview of the $\mathrm{NAD}^{+}$salvage pathways in Plasmodium parasites and the human host red blood cell. Plasmodium falciparum gene IDs and mammalian gene names are presented for each enzyme in italics. The red blood cell is shown in red, the parasitophorous membrane is shown in grey and the parasite membrane is shown in black. Na: nicotinic acid; Nam: nicotinamide; NR: nicotinamide ribotide; NaMN: nicotinate mononucleotide; NMN: nicotinamide mononucleotide; NaAD: nicotinate adenine dinucleotide; NAD ${ }^{+}$: nicotinamide adenine dinucleotide. NAPRT: nicotinic acid phosphoribosyltransferase, NMNAT: mononucleotide adenylyl transferase, NRK: nicotinamide riboside kinase, NP: nucleoside phosphorylase, NAMPT: nicotinamide phosphoribosyltransferase B. Live imaging of episomally expressed GFP tagged NAD ${ }^{+}$metabolic enzymes are shown (GFP-fusion proteins are shown in green). Enzyme Commission numbers are provided for each enzyme. All images are of trophozoite stage parasites. Hoechst dye (shown in blue) was used to visualize the parasite nucleus. *For example: sirtuins and poly(ADP-ribose) polymerases. doi:10.1371/journal.pone.0094061.g001

generate labeled $\mathrm{NADP}^{+}$, with only $7 \%$ containing any labeled ribose (Table 1).

To test whether the parasite could utilize both nicotinamide and nicotinic acid to drive $\mathrm{NAD}^{+}$synthesis, we performed media supplementation studies where each vitamin was provided at a standard RPMI concentration of $1 \mathrm{mg} / \mathrm{mL}$ or removed from the media to create a niacin free treatment. We observe that the parasite is able to take up and utilize both nicotinamide and nicotinic acid for $\mathrm{NAD}^{+}$synthesis, with $\mathrm{NAD}^{+}$levels increasing at the same rate for both forms of vitamin $B_{3}$ (nicotinamide: $\mathrm{d}\left[\mathrm{NAD}^{+}\right] / \mathrm{dt}=0.09 \pm 0.01, \quad$ nicotinic acid: $\mathrm{d}\left[\mathrm{NAD}^{+}\right] /$ $\mathrm{dt}=0.09 \pm 0.01)$ (Figure S4A in File S1). When niacin is removed from the media, $\mathrm{NAD}^{+}$levels remain low throughout development (Figure S4 in File S1). From this result we can confirm the lack of de novo $\mathrm{NAD}^{+}$synthesis, as it demonstrates that the production of $\mathrm{NAD}^{+}$is dependent on the availability of exogenous niacin. Therefore, during asexual development parasites are reliant on niacin uptake and can utilize both nicotinamide and nicotinic acid present in human serum to drive $\mathrm{NAD}^{+}$production via the PreissHandler pathway.

\section{Enzymatic activity of PfNMNAT}

To demonstrate that the predicted PfNMNAT (PF13_0159) was functional we established a discontinuous in vitro assay to measure the adenylyltransferase activity using purified recombinant His $6 \times-$ tagged protein (Figure 3A). PfNMNAT was found to have $\mathrm{a}_{\mathrm{m}}$ of $19.9 \pm 6.7 \mu \mathrm{M}$ for ATP and 35.8 $\pm 5.1 \mu \mathrm{M}$ for $\mathrm{NaMN}$, which is comparable to the $\mathrm{K}_{\mathrm{m}}$ constants reported for Bacillus anthracis (44 $\mu \mathrm{M}$ for ATP and $25 \mu \mathrm{M}$ for $\mathrm{NaMN}$ ) and other bacterial versions of this enzyme $[27,28]$. We also generated an active site mutant of PfNMNAT by replacing the conserved catalytic site residue Asp-110 with an alanine. This residue is thought to either hydrogen bond with the adenosine monophosphate during the adenylyltransferase reaction or help with essential $\mathrm{Mg}^{+2}$ coordination during catalysis [29,30]. The D110A mutant lacked in vitro activity in the enzyme assay confirming the essential function of this residue (data not shown). 

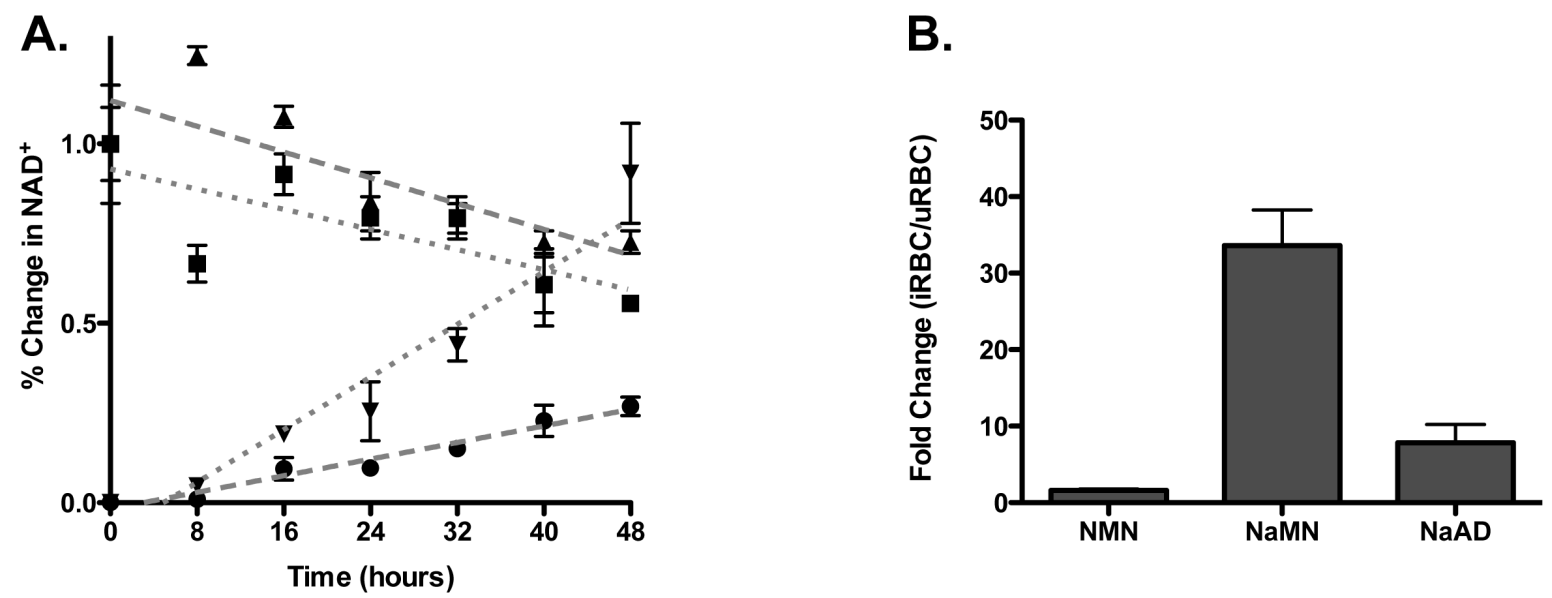

Figure 2. Characterization of $P$. falciparum NAD ${ }^{+}$metabolism. A. Time course of observed percent change in $N^{\prime} A D^{+}$levels in infected and

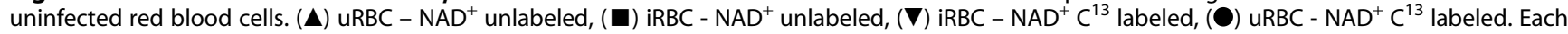
data point is normalized to the total pool of unlabeled and labeled NAD ${ }^{+}$present at time zero for the uRBC or iRBC. Error is reported as the standard deviation of three independent biological replicates. B. Fold Increase in Labeled NAD ${ }^{+}$Intermediates in iRBCs. The ratio represents the observed level of ${ }^{13} \mathrm{C}$-labeled intermediate observed in iRBC compared to that in uRBC after a 40 hour incubation with ${ }^{13} \mathrm{C}-\mathrm{U}$-glucose. Error is reported as the SD of three independent biological replicates. doi:10.1371/journal.pone.0094061.g002

\section{PfNMNAT complements NadD function in Escherichia coli}

Phylogenic analysis of PfNMNAT compared to representative prokaryotic and eukaryotic NMNATs demonstrates its similarity to bacterial versions of the enzyme (Figure 3B) [31]. Based on its high level of shared identity with the E. coli NMNAT (NadD) (Figure S5 in File S1), we tested whether PfNMNAT could genetically complement the essential bacterial enzyme in vivo [32]. We generated an E. coli strain where the nadD locus was replaced with the chloramphenicol (cam) drug resistance cassette (nadD::cam) and the non-essential linked gene, $y b e T$, was replaced with a kanamycin (kan) drug resistance cassette (ybe T::kan). P1 transduction was used to move nadD:: cam into acceptor strains carrying episomal copies of different NMNATs to test whether they could complement the deleted nadD. We selected transductants for the ybe T::kan marker and then subsequently screened for the nonselected nadD:::cam marker. Since $y b e T$ and nadD are linked, cam resistance should be observed in roughly $72 \%$ of the kan-resistant transductants when NadD function is supplied in trans; this is demonstrated in the positive control, in which $72 \%$ of kanresistant transductants exhibit cam resistance when $E$. coli nadD is provided episomally in the acceptor strain (Table 2). Using this approach we demonstrated that PfNMNAT is able to complement NadD, as $69 \%$ of transductants had cam resistance (Table 2). Additionally, as expected, the catalytically dead D110A version of PfNMNAT was unable to complement NadD function and linkage disruption was observed. Although five cam resistant transductants were obtained, sequencing subsequently confirmed that they contained suppressor mutations.

In order to validate our result, we tested whether PfNMNAT could rescue the nadD deletion strain of $E$. coli in a continuous growth assay. We placed an episomal copy of PfNMNAT into the nadD deletion strain under the control of the arabinose promoter, which is induced in the presence of arabinose and repressed in the presence of the competitive inhibitor fucose [33]. This strain was initially grown in the presence of arabinose and then back diluted into LB containing arabinose, fucose, or no inducer. When grown in the presence of the inducer arabinose, PfNMNAT is able to rescue growth more efficiently than in the no-inducer control (Figure S6 in File S1). When PfNMNAT expression is suppressed in presence of fucose, growth is not rescued and lags behind the control condition (Figure S6 in File S1). Taken together, both experimental approaches demonstrate the ability of PfNMNAT to complement E. coli $\mathrm{NadD}$. This demonstrates functional conser-

Table 1. Quantification of $\mathrm{NAD}^{+}$and $\mathrm{NADP}^{+}$levels in iRBCs.

\begin{tabular}{|c|c|c|}
\hline & \multicolumn{2}{|c|}{ concentration (uM) } \\
\hline & iRBC & uRBC \\
\hline NAD $^{+}$ & $880 \pm 100$ & $44 \pm 6$ \\
\hline \multirow[t]{3}{*}{ NADP $^{+}$} & $62 \pm 12$ & $35 \pm 1$ \\
\hline & \multicolumn{2}{|c|}{$\%$ of total pool } \\
\hline & iRBC & uRBC \\
\hline NADP $^{+}$-unlabeled & $93 \pm 7$ & $97 \pm 0.7$ \\
\hline NADP $^{+}-5{ }^{13} \mathrm{C}$ labeled & $3.5 \pm 2.4$ & $2.1 \pm 0.7$ \\
\hline NADP $^{+}-10{ }^{13} \mathrm{C}$ labeled & $3.5 \pm 5$ & $0 \pm 0$ \\
\hline
\end{tabular}




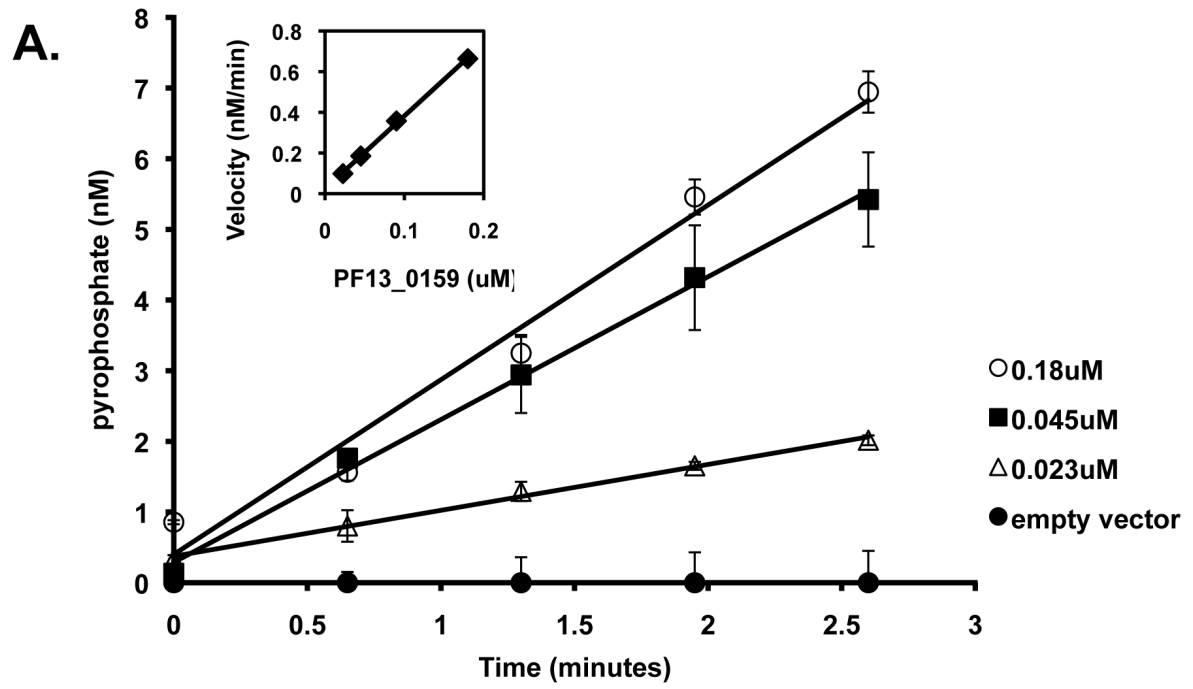

B.

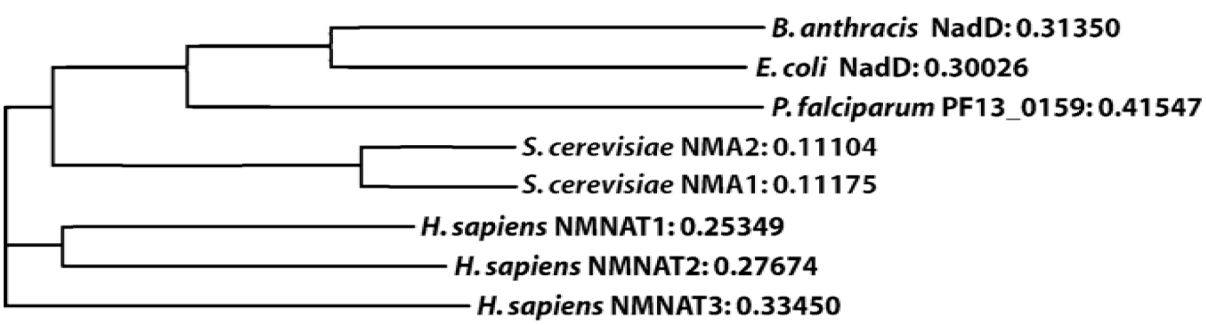

Figure 3. PF13_0159 encodes a nicotinate mononucleotide adenylyltransferase. A. Dependence of PF13_0159 adenylyltransferase activity on time and enzyme concentration. A discontinuous assay was established to measure enzyme activity; pyrophosphate release is measured as the end product of the adenylyltransferase reaction. Values were normalized to background absorbance values obtained in a buffer only control. A standard curve was generated to determine pyrophosphate concentrations. Error is reported at the SD of three independent technical replicates. B. Phylogenic analysis of PF13_0159 compared to representative prokaryotic and eukaryotic NMNATs. ClustalW2 was used to generate alignments and distance values are reported. An alignment of PF13_0159 and the E. coli NADD is shown in Figure S5 (in File S1).

doi:10.1371/journal.pone.0094061.g003

vation between the $P$. falciparum and $E$. coli enzymes and suggests that much of what is known about the well-characterized bacterial homologs of this enzyme may apply to PfNMNAT.

\section{NMNAT inhibitors disrupt Plasmodium falciparum growth in vivo}

Based on a previous report that demonstrated the anti-parasitic activity of the known bacterial NMNAT inhibitor, compound 1_03 [25], renamed la-a in this study, we sought to characterize the specificity of this compound further. Using our established enzymatic assay, we found that la-a exhibits specific activity against purified his-PfNMNAT (Figure 4A), and inhibits parasite growth in vivo with an $\mathrm{MIC}_{50}$ of $8.09 \pm 5.11 \mu \mathrm{M}$ (Figure $4 \mathrm{~B}$ ). When la-a is added to synchronous ring stage cultures at $50 \mu \mathrm{M}$, parasite growth arrests early in development (Figure 4C).

In order to attempt to improve certain features of the la-a scaffold design, we first developed an efficient chemical synthesis pathway to produce la-a. With a streamlined synthetic scheme in place, we utilized the chemical backbone of la-a (shown in Figure 5) to generate a number of derivatives to screen against live $P$. falciparum culture. Our approach was to improve the observed $\mathrm{MIC}_{50}$ of la-a derivatives by enhancing both their solubility and binding affinity. Solubility improvement was attempted principally by replacing hydrophobic groups with more hydrophilic moieties. Binding affinity was approached by estimating the points of likely enzyme-inhibitor interaction based on the co-crystal of the

Table 2. Complementation of E. coli NadD with PfNMNAT.

\begin{tabular}{|c|c|c|c|}
\hline \multirow[t]{2}{*}{ donor } & \multirow{2}{*}{ acceptor (episomal copy) } & \multirow{2}{*}{$\begin{array}{l}\text { selected marker } \\
\text { kan }\end{array}$} & \multirow{2}{*}{$\begin{array}{l}\text { unselected marker } \\
\text { cam }\end{array}$} \\
\hline & & & \\
\hline ybeT::kan & E. coli NadD & $100 / 100$ & $72 / 100$ \\
\hline \multirow[t]{3}{*}{ nadD::cam } & PfNMNAT wt & $100 / 100$ & $69 / 100$ \\
\hline & PfNMNAT D110A & $100 / 100$ & $5 / 100$ \\
\hline & empty vector & $100 / 100$ & $0 / 100$ \\
\hline
\end{tabular}

doi:10.1371/journal.pone.0094061.t002 

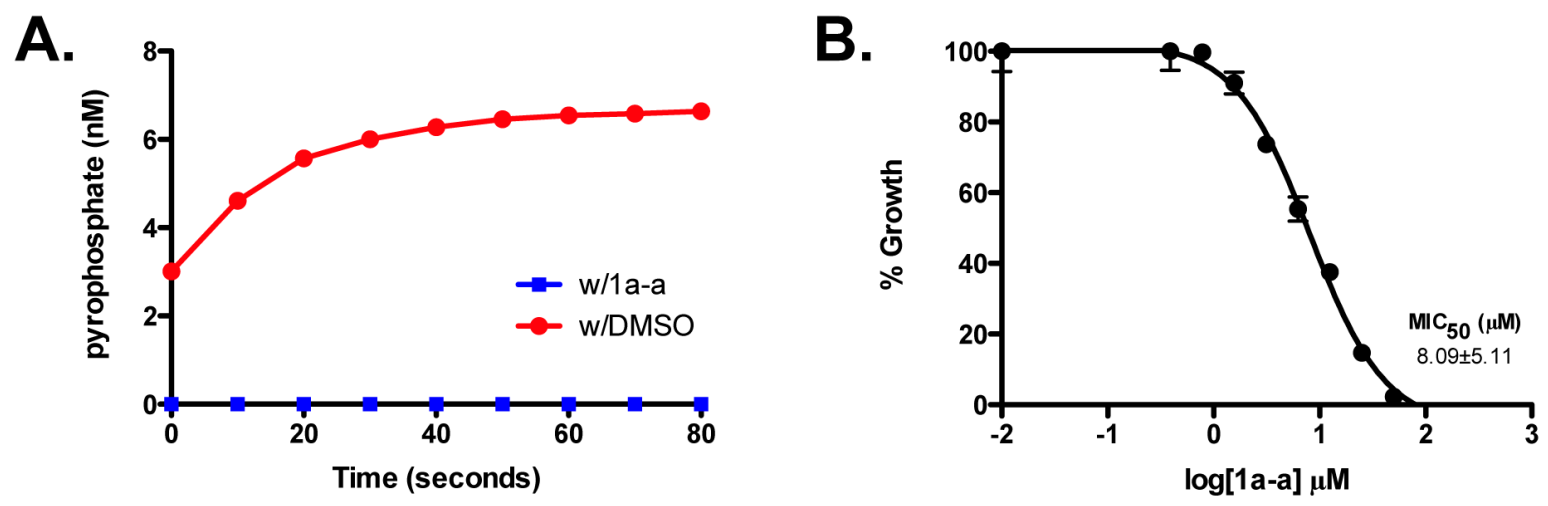

\section{C.}

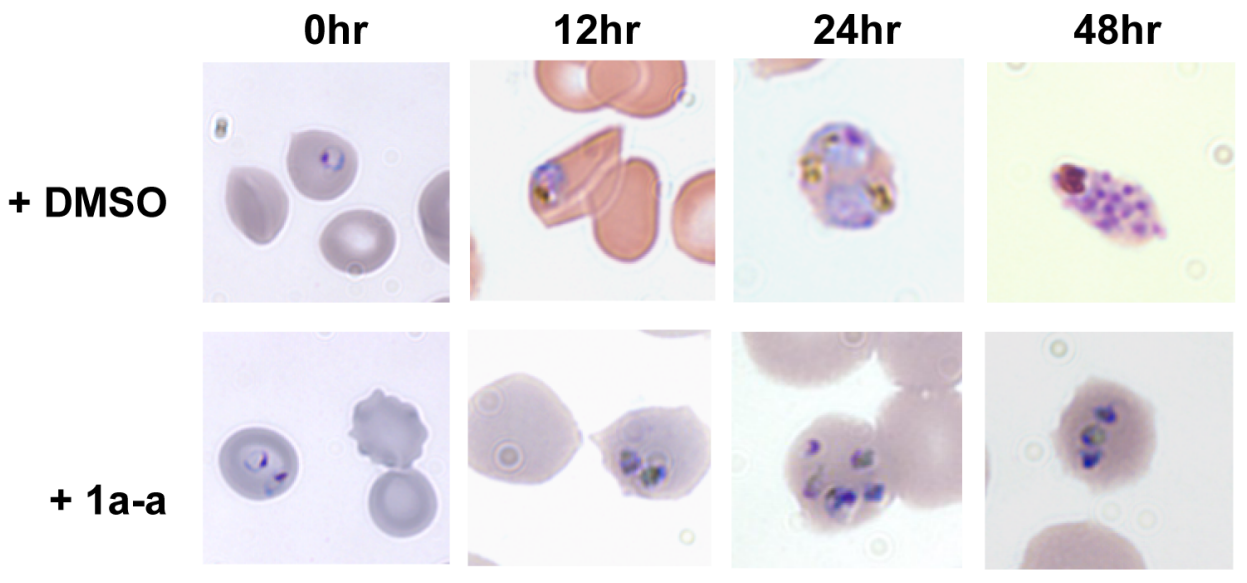

Figure 4. Inhibitory effect of putative NMNAT inhibitors in vitro. A. 1a-a inhibits adenylyltransferase activity of PfNMNAT. Purified enzyme was preincubated with either $50 \mu \mathrm{M} 1 \mathrm{a}-\mathrm{a}$ or $2 \%$ of DMSO for ten minutes before pyrophosphate release was monitored continuously at $565 \mathrm{~nm}$. A standard curve of pyrophosphate was used to determine concentration from observed absorbance values. Error is reported at the SD of three independent technical replicates. B. MIC 50 curve for 1a-a. A standard SYBR green growth assay was performed on synchronous ring stage parasites to determine $\mathrm{MIC}_{50}$ (See Material and Methods). Error is reported as the SD of three independent biological replicates. C. Imaging of $P$. falciparum treated with $50 \mu \mathrm{M}$ of 1a-a or a 1\% DMSO control. Parasite growth was monitored by imaging of fixed parasites visualized with Giemsa stain. doi:10.1371/journal.pone.0094061.g004

inhibitor bound to the bacterial NMNAT (PDB code MLA3) [34] and attempting to alter the spatial positioning at these points. To achieve these aims three regions of the molecular scaffold were modified and tested, the aniline moiety $\left(\mathrm{R}_{1}\right)$, the aromatic imine $\left(\mathrm{R}_{2}\right)$ and the central carbon chain $(\mathrm{n})$, resulting in over twenty novel compounds (Figure 5). All of the compounds were solubilized in dimethylsulfoxide (DMSO) and tested against live, synchronous $P$. falciparum 3D7 culture to determine experimental $\mathrm{MIC}_{50}$ concentrations, which are reported in Figure 5.

We chose to modify the aniline group $\left(\mathrm{R}_{1}\right)$ because differentially substituted anilines have previously been tested against bacterial NMNAT and have yielded strong inhibition (Sorci et al., 2009). The $\mathrm{MIC}_{50}$ values obtained for these substitutions (Figure 5) did not improve upon the lowest $\mathrm{MIC}_{50}$ of $8.09 \pm 5.11 \mu \mathrm{M}$ observed for the 4-bromoaniline substitution, therefore this $\mathrm{R}_{1}$ group was utilized in further compound development. We then tested a number of aromatic aldehydes at the $\mathbf{R}_{2}$ position, all imparting greater compound solubility than the anthracen-9-yl. These compounds were all effective in inhibiting parasite growth with the lowest $\mathrm{MIC}_{50}$ of $7.86 \mu \mathrm{M}$ observed for 2a-d (Figure 5).

The original la-a compound was determined to have a calculated $\log \mathrm{P}(\operatorname{cog} \mathrm{P})$ solubility index value of 5.835 (Figure 5).
Hydrophilic residues were incorporated into derivative compounds in an attempt to increase solubility (as reflected in lower clogP values) to obtain lower $\mathrm{MIC}_{50}$ values. The lowest $\operatorname{cog} \mathrm{P}$ value of 3.113 was obtained for compound $1 \mathrm{~g}$-e (Figure 5), with the highest $\operatorname{cog} \mathrm{P}$ value of 6.34 corresponding to the $2 \mathrm{a}-\mathrm{a}$. The trend of decreasing the $\operatorname{cog} \mathrm{P}$ values in the derivative compounds resulted in higher MIC $_{50}$ values, suggesting that these compounds were less effective in binding PfNMNAT.

We also made one modification to the carbon linker between the $R_{1}$ and $R_{2}$ groups with the addition of an extra carbon unit, with the prediction that this extension would allow for a better conformational fit in the pocket between the two active sites. Remarkably, the 2a-a compound differed from la-a only by the added carbon unit gave the greatest improvement with a $\mathrm{MIC}_{50}$ value of $0.708 \pm 2.35 \mu \mathrm{M}$ ) (Figure 5). Overall, six of the eight compounds where the carbon linker was modified had lower $\mathrm{MIC}_{50}$ values when the extra carbon unit was present (Figure 5). In summary, twenty different compounds were synthesized representing a wide range of functional group substitutions and clogP values. 


\begin{tabular}{|l|l|l|l|l|c|}
\hline \multicolumn{5}{|l|}{} \\
\hline ID & R1 & R2 & $\mathbf{n}$ & clogP* & MIC $_{50}(\boldsymbol{\mu M})$ \\
\hline 1a-a & 4-bromophenyl & anthracen-9-yl & 2 & 5.835 & $8.09 \pm 5.11$ \\
\hline 1a-c & 4-bromophenyl & 1,1'-biphenyl & 2 & 5.287 & $35.8 \pm 6.34$ \\
\hline 1a-d & 4-bromophenyl & 2,3-dimethoxynaphthalen-1-yl & 2 & 5.001 & $>100$ \\
\hline 1a-e & 4-bromophenyl & 1-methyl-1H-indol-3-yl & 2 & 4.264 & $32.5 \pm 5.61$ \\
\hline 1b-a & 3-bromophenyl & anthracen-9-yl & 2 & 5.811 & $26.3 \pm 14.3$ \\
\hline 1c-a & 2-bromophenyl & anthracen-9-yl & 2 & 5.787 & $>100$ \\
\hline 1d-a & 4-hydroxyphenyl & anthracen-9-yl & 2 & 4.547 & $65.9 \pm 54.5$ \\
\hline 1e-a & 3-hydroxyphenyl & anthracen-9-yl & 2 & 4.523 & $56.9 \pm 29.0$ \\
\hline 1f-a & 2-hydroxyphenyl & anthracen-9-yl & 2 & 4.759 & $>100$ \\
\hline 1g-a & 4-fluorophenyl & anthracen-9-yl & 2 & 5.190 & $71.7 \pm 69.7$ \\
\hline 1g-c & 4-fluorophenyl & 1,1'-biphenyl & 2 & 4.642 & $40.2 \pm 6.77$ \\
\hline 1g-d & 4-fluorophenyl & 2,3-dimethoxynaphthalen-1-yl & 2 & 3.850 & $91.7 \pm 28.9$ \\
\hline 1g-e & 4-fluorophenyl & 1-methyl-1H-indol-3-yl & 2 & 3.113 & $>100$ \\
\hline 2a-a & 4-bromophenyl & anthracen-9-yl & 3 & 6.340 & $0.708 \pm 2.35$ \\
\hline 2a-c & 4-bromophenyl & 1,1'-biphenyl & 3 & 5.793 & $22.7 \pm 4.01$ \\
\hline 2a-d & 4-bromophenyl & 2,3-dimethoxynaphthalen-1-yl & 3 & 5.001 & $7.86 \pm 6.61$ \\
\hline 2a-e & 4-bromophenyl & 1-methyl-1H-indol-3-yl & 3 & 4.264 & $34.1 \pm 15.0$ \\
\hline 2g-a & 4-fluorophenyl & anthracen-9-yl & 3 & 5.695 & $>100$ \\
\hline 2g-c & 4-fluorophenyl & 1,1'-biphenyl & 3 & 5.147 & $12.9 \pm 6.90$ \\
\hline 2g-d & 4-fluorophenyl & 2,3-dimethoxynaphthalen-1-yl & 3 & 4.365 & $38.5 \pm 27.2$ \\
\hline 2g-e & 4-fluorophenyl & 1-methyl-1H-indol-3-yl & 3 & 3.618 & $49.1 \pm 35.7$ \\
\hline
\end{tabular}

Figure 5. Antimalarial activity and partition coefficients for putative NMNAT inhibitors. MIC 50 values were determined for each synthesized NMNAT inhibitor using a standard SYBR green growth assay on synchronous ring stage parasites. Error is reported as the SD of three independent biological replicates. ${ }^{*}$ Calculated octanol/water partition coefficients (cLogPs) for all compounds were determined using online tools at http://www.molinspiration.com.

doi:10.1371/journal.pone.0094061.g005

\section{Discussion}

In this study, we utilized various experimental approaches to characterize the $\mathrm{NAD}^{+}$biosynthetic pathway of Plasmodium falciparum. Bioinformatic analysis identified the components of a canonical salvage pathway in the parasite, but did not identify the enzymes involved in de novo synthesis, suggesting that the parasite is a $\mathrm{NAD}^{+}$auxotroph [24]. To confirm the architecture of the parasite's $\mathrm{NAD}^{+}$synthetic pathway, stable isotope labeling via fully labeled ${ }^{13} \mathrm{C}$-U-glucose was used to trace labeled carbon into newly synthesized $\mathrm{NAD}^{+}$throughout development. This demonstrated the steady and continuous synthesis of $\mathrm{NAD}^{+}$throughout the 48hour parasite life cycle as well as showing a steady rate of turnover of unlabeled $\mathrm{NAD}^{+}$. Both the rate of synthesis and total concentration of $\mathrm{NAD}^{+}$are much higher in infected red blood cells when compared to uninfected control cultures. Coupled with the observation of increased levels of labeled $\mathrm{NaMN}$ and $\mathrm{NaAD}$ in iRBCs, we can conclude that the high levels of $\mathrm{NAD}^{+}$are being synthesized and utilized by the parasite and not the host cell during infection. This is finding is supported by previous reports of elevated level of $\mathrm{NAD}^{+}$in infected erythrocytes and the observation that nicotinamidase activity, an enzyme found in the parasite and not in the host, is increased in P. falciparum infected red blood cells [9]. The measured intracellular $\mathrm{NAD}^{+}$concentrations are also in agreement with those reported in a NMR spectroscopy study of purified parasites and uninfected RBCs, which also found higher $\mathrm{NAD}^{+}$levels in isolated trophozoites [35]. Elevated $\mathrm{NAD}^{+}$synthesis driven by the parasite suggests that the $\mathrm{NAD}^{+}$pathway can be pursued as a viable therapeutic target without compromising host erythrocyte metabolism.

Despite a ten-fold difference in $\mathrm{NAD}^{+}$levels, we observe little difference in the concentration of $\mathrm{NADP}^{+}$between iRBCs and uRBCs. Even more striking is the small percentage of the newly synthesized $\mathrm{NAD}^{+}$that becomes phosphorylated. Although a putative $\mathrm{NAD}^{+}$kinase (PFI0650c, EC 2.7.1.23) and pyridine nucleotide $\left(\mathrm{NADP}^{+}\right)$transhydrogenase (PF14_0508, EG 1.6.1.2) have been identified, they have not been characterized. NADPH is utilized by both the glutathione and thioredoxin systems in the parasite to deal with oxidative stress [36]. It is additionally utilized in a number of anabolic pathways, such as isoprenoid and fatty acid synthesis $[37,38]$. In all of these processes, $\mathrm{NADP}^{+}$is cycled 
between its oxidized and reduced states without being consumed. It is therefore possible that the amount of $\operatorname{NADP}(\mathrm{H})$, while much less than the total pool of $\mathrm{NAD}^{+}$, is sufficient for blood stage development.

Gene expression data $[39,40]$ for the $\mathrm{NAD}^{+}$metabolic enzymes have suggested that they have unusual and uncoordinated bi- and mono-phasic transcriptional profiles. The parasite nicotinamidase and NMNAT exhibit transcriptional peaks around 20 and 40 hours post invasion, while the nicotinate phosphoribosyltransferase and $\mathrm{NAD}^{+}$synthase enzymes are maximally transcribed between 25-28 hours. Although it has been proposed [41] that $\mathrm{NAD}^{+}$synthesis would peak with $\mathrm{NAD}^{+}$synthase expression, we observe steady $\mathrm{NAD}^{+}$production throughout the IDC. This is likely necessary to maintain $\mathrm{NAD}^{+}$pools to replace the $\mathrm{NAD}^{+}$that is lost to turnover and provides a sufficient increase in total $\mathrm{NAD}^{+}$ required for the development of new merozoites.

We were able to confirm the parasite's reliance on uptake of exogenous niacin in order to synthesize $\mathrm{NAD}^{+}$via the canonical Preiss-Handler salvage pathway. When niacin was removed from the culture media, intracellular concentrations of $\mathrm{NAD}^{+}$were significantly lower. However, in agreement with previous nutrient limitation studies, niacin deprivation did not lead to a growth defect in vitro suggesting that the niacin provided by the host cell is sufficient to provide the required amount of $\mathrm{NAD}^{+}$synthesis necessary for growth in vitro [42]. This is unsurprising during the asexual and sexual stages in the human host, where the parasite is developing in the niacin rich environments of human hepatocytes, red blood cells and serum [43]. Furthermore, a reliance on niacin salvage has been shown for other pathogenic organisms, such as Leishmania infantum and multiple species of pathogenic yeast, making it a common evolutionary adaptation $[44,45]$. In multiple species of yeast, niacin transporters have been characterized and shown to be upregulated in niacin starvation conditions $[46,47]$. To date, however, no homologs of these identified transporters have been identified in the Plasmodium genome. The parasite's reliance on a salvage pathway and dependence on the availability of niacin makes it increasingly susceptible to multiple points of interventions.

NMNATs have emerged as an attractive drug target due to their indispensible role in $\mathrm{NAD}^{+}$synthesis, catalyzing the essential step shared by both the de novo and salvage pathways [48,49]. Our biochemical characterization of PfNMNAT validates its function as a bona fide nicotinate mononucleotide adenylyltransferase and demonstrates the essentiality of the conserved active site Asp110 residue for its catalytic activity. Proteomic data has detected the presence of PfNMNAT peptides during both the asexual and sexual stages further supporting its role in these life stages and suggesting that potential inhibitors could target both important life stages [50,51]. Furthermore, PfNMNAT is well conserved across all species of Plasmodium as well as in other parasitic organisms in the Apicomplexan phylum such as Babesia bovis and Theileria annulata, [52,53] implicating NMNATs as a drug target beyond $P$. falciparum.

In this study, we demonstrated the high level of conservation between the parasite NMNAT and its bacterial homolog by complementing the essential E. coli $\mathrm{NadD}$ in vivo. This similarity is striking when compared to the high level of divergence between the parasite and the three human NMNATs as demonstrated by an alignment of the four protein sequences (Figure S7 in File S1). Previous comparison of the solved $E$. coli and human NMNATs crystal structures has shown significant structural divergence between their respective active sites, suggesting the ability to selectively target the bacterial enzymes [26]. Due to both the similarity of the parasite PfNMNAT to its bacterial homologs and its divergence from the host enzyme, it presents a new target for chemical inhibition. Inhibitors of bacterial NMNATs have been previously identified based on in silico screening of databases of compounds against solved three-dimensional protein structures. These compounds were then further tested both in vitro and in vivo to identify lead compounds for drug development and select for those that demonstrated no inhibitory effect on the human NMNATs [26,34]. A structure of a co-crystal of the bacterial NMNAT and one of the most effective inhibitors, compound la-a, suggested that this compound and those closely related to it, bind to the enzyme in its apo form and interact with both the NaMN binding pocket and the ATP binding site [34]. Since both regions are conserved in PfNMNAT we reasoned that such inhibitors might interact with the parasite enzyme in a similar fashion and prove effective in inhibiting growth in vivo, without impacting host metabolism.

Starting with the chemical backbone of the original la-a compound, we made a number of modifications to generate the derivatives tested in this study. We attempted to increase compound solubility, as reflected in lower $\operatorname{cog} \mathrm{P}$ values, in order to increase drug potency. We synthesized compounds in a range of clogP values from 6.34-3.113 (Figure 5), which resulted in the opposite effect, with the highest $\log \mathrm{P}$ values corresponding to the lowest $\mathrm{MIC}_{50}$ values. This could be the result of a number of different factors such as a hydrophobic effect on binding, disruption of important hydrogen bonds within the active site and other steric effects.

Multiple modifications were made to the $\mathrm{R}_{1}$ group. In Huang et al., a solved structure with la-a and the $B$. anthracis NMNAT demonstrated an interaction between the para positioned bromine and the His 18 residue in the T/HXGH motif. None of our $\mathrm{R}_{1}$ modifications resulted in significant improvement over the $\mathrm{MIC}_{50}$ value of $1 \mathrm{a}-\mathrm{a}$, suggesting that the para positioned bromine allows for the best interaction with the ATP binding site. The polyaromatic moiety present as the $\mathrm{R}_{2}$ group in la-a was shown by crystallography to exhibit $\pi$-stacking with up to three aromatic residues of the $B$. anthracis NMNAT [34] in the nicotinosyl binding pocket. To optimize this effect, a number of aromatic aldehydes were tested at the $\mathrm{R}_{2}$ position. These moieties imparted greater compound solubility as reflected by their lower calculated partition coefficient $(\operatorname{cog} \mathrm{P})$ values, but were not found to significantly lower $\mathrm{MIC}_{50}$ values (Figure 5). The addition of an extra carbon unit to the linker between the $R_{1}$ and $R_{2}$ groups was predicted to allow for a better conformational fit in the pocket between the two active sites. This single modification is the sole difference between the 2aa and la-a compounds and resulted in almost a ten-fold improvement in its $\mathrm{MIC}_{50}$ value (Figure 5). Overall, this modification presented the best improvement to potency obtained for the compounds generated and tested in this limited study, even though many of the synthesized compounds exhibited modest antimalarial activity. Without a solved crystal structure of PfNMNAT it is hard to correlate $\mathrm{MIC}_{50}$ with the specific features of compound structure, especially the novel functional groups utilized in our study. Moving forward it will be essential to further characterize both the PfNMNAT enzyme and explore further chemical modifications to this identified compound scaffold in order to generate viable compounds for drug development. Additionally, any identified lead compounds will need to be screened for potential activity against the human NMNATs to ensure they do not display cross-reactivity.

In summary, this work highlights $\mathrm{NAD}^{+}$metabolism as a new area of interest in Plasmodium biology and potentially other closely related Apicomplexan organisms. The identification of both novel drug targets and putative lead compounds is significant at a time 
when drug resistance is on the rise and few lead antimalarial compounds are in later stages of drug development.

\section{Materials and Methods}

\section{$P$. falciparum Culture}

The P. falciparum 3D7 and D10 clones were cultured and synchronized by standard methods $[54,55]$ with the modification that culture flasks were maintained at $37^{\circ}$ in an atmospherically controlled incubator set at 5\% $\mathrm{CO}_{2}, 6 \% \mathrm{O}_{2}$. Synchronicity and parasitemia was monitored using microscopy and Giemsa stained smears. P. falciparum strains used in this study were obtained from the Malaria Research and Reference Reagent Resource Center (MR4, www.mr4.org), which is a part of the NIAID BEI Resources collection.

For niacin deprivation experiments standard RPMI media [56] was reconstituted using 50× RPMI Amino Acid Solution (Sigma Aldrich) and individual vitamins and salts purchased from Sigma Aldrich and Fisher Chemical. Fully reconstituted RPMI was always made in parallel and used as a control to ensure there was no variation in parasite growth between batches.

\section{Cellular localization of GFP-tagged $\mathrm{NAD}^{+}$metabolism enzymes}

The four full length gene loci corresponding to the enzymes of the Plasmodium $\mathrm{NAD}^{+}$salvage pathway (pf13_0159, pff1410c, $p f i 1310 w, p f c 0910 c)$ were cloned into the pDC2-CAM-CRT-GFP plasmid [57] to create C-terminal GFP fusions under the control of the P. falciparum calmodulin promoter (pf14_0323) using the F and R pDC2 (Table $\mathrm{S} 1$ in File S1). These plasmids were transfected via electroporation into ring stage D10 culture with a BioRad GenePulser and maintained episomally under WR99210 selection [58]. Live parasites were stained with Hoechst dye to visualize the nucleus and then imaged with a Leica microscope equipped with SlideBook software to determine the localization of the protein-GFP fusions. Protein-GFP fusions were also validated by Western blot. Lysates were prepared from mixed stage culture and separated on 10\% denaturing Tris-glycine gels. Lysates were transferred to nitrocellulose membrane using the wet transfer method overnight at $4^{\circ}, 20 \mathrm{~V}$ and probed with Roche anti-GFP antibody (11 814460001$)$ at 1:1500, $1 \mathrm{~h}$, followed by probing with Sigma anti-rat IgG (whole molecule) peroxidase conjugate (A 9037) at 1:2000, $45 \mathrm{~min}$. The signal was visualized using Pierce ECL reagent.

\section{LC-MS/MS detection of NAD ${ }^{+}$intermediates}

The $P$. falciparum 3D7 clone was cultured under standard conditions at a starting parasitemia of $6 \%$ and hematocrit of $2 \%$. Isotopic labeling was initiated by resuspending cultures in RPMI containing ${ }^{13} \mathrm{C}$-U-glucose. A parallel uninfected erythrocyte culture (from the same blood donor) was treated identically and samples were collected every 8 hours from both $\mathrm{RBC}$ and $\mathrm{URBC}$ cultures. Metabolomic extraction, LC-MS/MS instrumentation, and data extraction was performed as described previously [7]. Briefly, cultures were centrifuged and the cell pellet was extracted first into 4 volumes of $100 \%$ methanol on dry ice and then with one volume of ice-cold 80:20 methanol:water in a water bath sonicator. Samples were dried down under nitrogen and resuspended in water prior to analysis. LC-MS/MS was performed using a Shimadzu LC-10A HPLC system and a Phenomenex Luna aminopropyl column $(250 \mathrm{~mm} 3 \times 2 \mathrm{~mm}$ with a $5 \mathrm{~mm}$ particle size) coupled to a Finnigan TSQ Quantum Ultra triple quadrupole mass spectrometer (Thermo Electron Corporation) running in positive mode. Data was collected and processed by Xcalibur software (Thermo Electron Corporation). All data is representative of the standard deviation of three biological replicates.

To determine the intracellular concentration of $\mathrm{NAD}^{+}$and $\mathrm{NADP}^{+}$two methods were taken. Absolute quantification was performed via ${ }^{13} \mathrm{C}$-labeling for 72-hours as described in (Bennett et al., 2008). Once the intracellular metabolite pools were close to completely ${ }^{13} \mathrm{C}$-labeled, metabolites were extracted and spiked with unlabeled $\mathrm{NAD}^{+}$and $\mathrm{NADP}^{+}$in a dilution series. Unlabeled $\mathrm{NAD}^{+}$and $\mathrm{NADP}^{+}$standard curves were produced and the biological $\left({ }^{13} \mathrm{C}\right.$-labeled) $\mathrm{NAD}^{+}$and $\mathrm{NADP}^{+}$concentrations were determined using a linear fit. Final intracellular concentrations were determined by determining the total cell number via hemocytometer cell counting and assuming the intracellular volume of an infected red blood cell is $75 \mathrm{fL}$ [59]. The second approach was measuring the intracellular $\mathrm{NAD}^{+}$and $\mathrm{NADP}^{+}$ under unlabeled conditions. Four identical samples from each iRBC and URBC cultures were taken and subsequently spiked in with different concentrations of unlabeled $\mathrm{NAD}^{+}$and $\mathrm{NADP}^{+}$. Total ion counts were collected in the dynamic range via positive mode LC-MS and the cellular background subtracted from the spike-in samples. A standard curve was constructed and intracellular concentration determined as described above.

\section{Purification of PfNMNAT recombinant protein}

The pf13_0159 open reading frame (615 bp) was cloned into pProEX (Invitrogen) in order to generate a N-terminal $6 \times$ HIS tag fusion using the $p f 13 \_0159$ pProEX F and R primers (Table S1 in File S1). Recombinant protein was expressed in Escherichia coli BL21 (RIL Codon PLUS) (Stratagene) cells grown at $25^{\circ}$ and induced with $0.5 \mathrm{mM}$ Isopropyl $\beta$-D-1-thiogalactopyranoside (IPTG) overnight. The induced culture was pelleted, resuspended in buffer $(20 \mathrm{mM}$ imidazole in $1 \times$ PBS $)$ and then mechanically lysed. Protein was purified over Ni Sepharose 6 Fast Flow resin (GE Healthcare) and eluted with $500 \mathrm{mM}$ imidazole phosphate buffer. Purity of the $26 \mathrm{kDa}$ fusion protein was verified by silver stain SDS/PAGE.

To generate the PfNMNAT D110A mutation, site directed mutagenesis was used. The wild type PF13_0159 locus was subcloned into the StrataClone vector (Agilent Technologies) and PCR was performed using the overlapping primers, D110A SENSE and ANTISENSE (Table S1 in File S1), containing the desired mutation. To recover the mutated plasmid, the native template was digested with DpnI (New England Biolabs) and then transformed into E. coli strain DH5 $\alpha$.

\section{PfNMNAT enzymatic assays}

Prior to assays, purified PfNMNAT- $6 \times$ HIS protein was dialyzed into reaction buffer containing $100 \mathrm{mM}$ HEPES and $10 \mathrm{mM} \mathrm{MgCl}_{2}$ at $\mathrm{pH} 7.5$ overnight at $4^{\circ}$ using $3500 \mathrm{Da}$ MWCO dialysis tubing from Fisherbrand. A discontinuous assay was set up to determine the steady-state kinetic parameters for PfNMNAT as previously described [26]. This approach couples the release of the byproduct pyrophosphate to the colorimetric detection of free phosphate.

(1) NaMN adenylyltransferase reaction: $\mathrm{NaMN}+\mathrm{ATP} \rightarrow$ $\mathrm{NaAD}^{+}+\mathrm{PP}_{\mathrm{i}}$

(2) Inorganic pyrophosphatase reaction: $\mathrm{PP}_{\mathrm{i}}+\mathrm{H}_{2} \mathrm{O} \rightarrow 2 \mathrm{P}_{\mathrm{i}}$

Pyrophosphate release was visualized using the $\mathrm{P}_{\mathrm{i}}$ Per Pyrophosphate Assay Kit (Invitrogen). $100 \mu \mathrm{L}$ reactions were set up in 96well plates (BD Biosciences) according to manufacturer specifications. Nicotinic acid mononucleotide was obtained at maximum 
purity from Sigma Aldrich. ATP was purchased from Fisher Bioreagents. Steady-state kinetic analysis of PfNMNAT was performed by varying each substrate concentration (NaMN and ATP) in a range from 0 to $500 \mu \mathrm{M}$, while providing the other substrate at $500 \mu \mathrm{M}$. Assays were monitored continuously at $565 \mathrm{~nm}$ using a BioTek Synergy MX plate reader equipped with Gen 5 software. To subtract for any background activity present in the active fraction, an empty vector control was purified following the same protocol. Apparent $\mathrm{K}_{\mathrm{m}}$ values were calculated by fitting the data to a standard Michaelis-Menten model using GraphPad Prism Version 5.0 software.

\section{Genetic complementation of the $E$. coli NadD with PfNMNAT}

The nadD locus from E. coli (642 bp) was cloned into pBAD18 $[33,60]$ using the ecNadD pBAD $\mathrm{F}$ and $\mathrm{R}$ primers (Table $\mathrm{S} 1$ in File $\mathrm{Sl}$ ) to generate $\mathrm{pNadD}$, which was transformed into the $E$. coli strain MC4100 containing the linked ybe T::cam marker (strain JO1, Table S2 in File S1). Chromosomal nadD was replaced by a kanamycin resistance cassette amplified using the primers CamKanNadD F and R primers (Table $\mathrm{S} 1$ in File $\mathrm{S} 1$ ) in the $E$. coli strain DY378 (Yu et al., 2000) containing pNadD by lambda Red recombineering [61]. The resulting nadD::kan knockout strain (JO2, Table S2 in File S1) was used as a donor for P1-mediated transduction [62]. Designated acceptor strains contained episomal copies of either E. coli nadD or PfNMNAT, and transductants were selected on LB agar containing kanamycin and appropriate inducer (L-arabinose or IPTG). Growth assays were conducted in 96 well plates and continuously monitored for absorbance at $600 \mathrm{~nm}$ using a BioTek Synergy MX plate reader equipped with Gen5 software for 10 hours. E. coli containing pBAD18::PfNMNAT were grown in the presence of arabinose to late log phase and then diluted into $\mathrm{LB}$ or LB containing $0.2 \%(\mathrm{~g} / \mathrm{mL})$ arabinose or $0.05 \%(\mathrm{~g} / \mathrm{mL})$ fucose.

\section{Synthesis of NMNAT inhibitors}

All reactions were carried out under an inert atmosphere of nitrogen unless otherwise indicated. Tetrahydrofuran (THF) and dichloromethane (DCM) were purified by passage of the argonpurged solvents through activated alumina columns. Other commercial reagents were used as received, without further purification. Reactions were monitored by thin layer chromatography (TLC) performed on $0.25 \mathrm{~mm}$ Whatman silica gel places (60 A) with fluorescent indicator using UV light as a visualizing agent. The plates were subsequently developed using basic potassium permanganate, ceric ammonium molybdate or $p$ anisaldehyde stain.

Nuclear magnetic resonance (NMR) spectra were obtained on $500 \mathrm{MHz}$ Bruker AVANCE and Varian Unity/INOVA spectrometers. ${ }^{1} \mathrm{H}$ NMR spectra are referenced to the residual monoprotio-solvent peak ((GD $\left.)_{2} \mathrm{SO}: 2.50 \mathrm{ppm}\right)$ and ${ }^{13} \mathrm{C} \mathrm{NMR}$ are referenced to the deuterated solvent signal $\left(\left(\mathrm{CD}_{3}\right)_{2} \mathrm{SO}\right.$ : $39.52 \mathrm{ppm})$. Multiple shifts arriving from conformational isomers are separated by a slash (shift $1 /$ shift 2). Multiplicities are abbreviated as follows: $\mathrm{s}=$ singlet, $\mathrm{d}=$ doublet, $\mathrm{t}=$ triplet, $\mathrm{q}=$ quartet, $\mathrm{m}=$ multiplet, $\mathrm{br}=$ broad signal. High-resolution mass spectra were obtained on an Agilent 6210 High-Resolution Time-of-Flight mass spectrometer coupled to an Agilent Technologies 1200 series High Performance Liquid Chromatography system using no

\section{References}

1. Miller LH, Ackerman HC, Su XZ, Wellems TE (2013) Malaria biology and disease pathogenesis: insights for new treatments. Nat Med 19: 156-167. doi: $10.1038 / \mathrm{nm} .3073$.
HPLC column. Details specific to the synthesis of each compound are presented in the Supporting Info.

\section{P. falciparum growth inhibition assays with PfNMNAT inhibitors}

Growth inhibition assays were conducted as standard SYBR green I fluorescence assays [63] with the noted alterations. $200 \mu \mathrm{L}$ aliquots of $0.5 \%$ synchronous ring stage culture at $2 \%$ hematocrit were set up in 96-well plates and incubated for 72 hours at standard culture conditions with varying concentrations of drug dissolved in DMSO. NMNAT inhibitors were resuspended to make $10 \mathrm{mM}$ stock solutions. After growth, plates were placed at $-80^{\circ}$ overnight and thawed at room temperature. Once lysed, $100 \mathrm{uL}$ of culture was incubated with SYBR green I dye (Sigma) $(0.4 \mu \mathrm{L} / \mathrm{mL})$ in $100 \mu \mathrm{L}$ of buffer $(20 \mathrm{mM}$ Tris-HCl, pH 7.5; 5 mM EDTA; $0.08 \%$ Triton X-100; 0.008\% saponin). Fluorescence was quantified (excitation: $485 \mathrm{~nm}$; emission: $535 \mathrm{~nm}$ ) using a BioTek Synergy MX plate reader equipped with Gen5 software. Assays were always run in parallel with a chloroquine treated control growth assay.

\section{Supporting Information}

File S1 Contains the files: Figure S1. PfNico (PFC0910w) localization throughout the IDG. Live imaging of episomally expressed enzyme-GFP fusion proteins in the parasite. Figure S2. Labeling pattern of $\mathbf{N A D}^{+}$. The resulting labeling pattern of NAD+ from C13-U-glucose is depicted. Figure S3. Observed labeling pattern of NAD $^{+}$in iRBCs. Comparison of half and full labeled NAD+ generated in iRBCs in the presence of C13-Uglucose. Figure $\mathrm{S4}$. NAD ${ }^{+}$synthesis under different niacin conditions. Observed fold change in NAD+ concentration resulting from different niacin present in the culture medium. Figure S5. Alignment of PfNMNAT and the $E$. coli homolog NadD. Comparison of the amino acid sequences of the parasite and bacterial NMNAT enzymes. Figure S6. Complementation of $\boldsymbol{E}$. coli NadD with PfNMNAT. The ability of the parasite NMNAT to rescue $E$. coli growth is demonstrated in a continuous growth assay. Figure S7. Alignment of PfNMNAT and the Human NMNAT homologs. Comparison of the amino acid sequences of the parasite and human NMNAT enzymes. Table S1. Primers Used in This Study. Table S2. Strains Used in This Study.

(PDF)

\section{Acknowledgments}

We would like to thank $\mathrm{H}$. Zhang and N. Huang for helpful discussions throughout this project as well as J. Morrissey and members of the Llinás lab for their advice and technical assistance on this work. We thank D. Ricci and T. Silhavy for the MC4100 E. coli strain and assistance with the NadD complementation. We also thank H. Ginsburg, J. Santos, and H. Painter for critical reading of this manuscript.

\section{Author Contributions}

Conceived and designed the experiments: JKO SC PJR ML. Performed the experiments: JKO LJK SC JT TAB. Analyzed the data: JKO TAB PJR ML. Contributed reagents/materials/analysis tools: PJR ML. Wrote the paper: JKO TAB ML.

2. Sachs J, Malaney P (2002) The economic and social burden of malaria. Nature 415: 680-685. doi:10.1038/415680a. 
3. WHO (2011) The World Malaria Report. Geneva: World Health Organization. Available: http://www.who.int/malaria/world_malaria_report_2011/en/ index.html.

4. Roth E (1990) Plasmodium falciparum carbohydrate metabolism: a connection between host cell and parasite. Blood Cells 16: 453-60; discussion 461-6.

5. Roth EF, Raventos-Suarez C, Perkins M, Nagel RL (1982) Glutathione stability and oxidative stress in P. falciparum infection in vitro: responses of normal and G6PD deficient cells. Biochem Biophys Res Commun 109: 355-362.

6. Nagarajan K (1964) Pyruvate and Lactate Levels in Relationship to the Nicotinamide-Adenine Dinucleotide Levels in Malarial Parasites (Plasmodium Berghei). Biochim Biophys Acta 93: 176-179.

7. Olszewski KL, Morrisey JM, Wilinski D, Burns JM, Vaidya AB, et al. (2009) Host-parasite interactions revealed by Plasmodium falciparum metabolomics. Cell Host Microbe 5: 191-199. doi:10.1016/j.chom.2009.01.004.

8. Sherman IW (1966) Levels of oxidized and reduced pyridine nucleotides in avian malaria (Plasmodium lophurae). Am J Trop Med Hyg 15: 814-817.

9. Zerez CR, Roth EF, Schulman S, Tanaka KR (1990) Increased nicotinamide adenine dinucleotide content and synthesis in Plasmodium falciparum-infected human erythrocytes. Blood 75: 1705-1710.

10. Harden A, Young W (1906) The Alcoholic Ferment of Yeast-Juice. Proceedings of the Royal Society of London 78: 369-375.

11. Billington RA, Bruzzone S, De Flora A, Genazzani AA, Koch-Nolte F, et al. (2006) Emerging functions of extracellular pyridine nucleotides. Mol Med 12: 324-327. doi: 10.2119/2006'Äi00075.Billington.

12. Nakamura M, Bhatnagar A, Sadoshima J (2012) Overview of pyridine nucleotides review series. Circ Res 111: 604-610. doi:10.1161/CIRCRESAHA.111.247924.

13. Gardner MJ, Hall N, Fung E, White O, Berriman M, et al. (2002) Genome sequence of the human malaria parasite Plasmodium falciparum. Nature 419: 498-511. doi:10.1038/nature01097.

14. North BJ, Verdin E (2004) Sirtuins: Sir2-related NAD-dependent protein deacetylases. Genome Biol 5: 224. doi:10.1186/gb-2004-5-5-224.

15. Duraisingh MT, Voss TS, Marty AJ, Duffy MF, Good RT, et al. (2005) Heterochromatin silencing and locus repositioning linked to regulation of virulence genes in Plasmodium falciparum. Cell 121: 13-24. doi:10.1016/ j.cell.2005.01.036.

16. Freitas-Junior LH, Hernandez-Rivas R, Ralph SA, Montiel-Condado D, Ruvalcaba-Salazar OK, et al. (2005) Telomeric heterochromatin propagation and histone acetylation control mutually exclusive expression of antigenic variation genes in malaria parasites. Cell 121: 25-36. doi:10.1016/ j.cell.2005.01.037.

17. Mancio-Silva L, Lopez-RubioJJ, Glaes A, Scherf A (2013) Sir2a regulates rDNA transcription and multiplication rate in the human malaria parasite Plasmodium falciparum. Nat Commun 4: 1530. doi:10.1038/ncomms2539.

18. Tonkin CJ, Carret CK, Duraisingh MT, Voss TS, Ralph SA, et al. (2009) Sir2 paralogues cooperate to regulate virulence genes and antigenic variation in Plasmodium falciparum. PLoS Biol 7: e84. doi:10.1371/journal.pbio.1000084.

19. Bogan KL, Brenner C (2008) Nicotinic acid, nicotinamide, and nicotinamide riboside: a molecular evaluation of $\mathrm{NAD}+$ precursor vitamins in human nutrition. Annu Rev Nutr 28: 115-130. doi:10.1146/annurev.nutr.28.061807. 155443.

20. Preiss J, Handler P (1958) Biosynthesis of diphosphopyridine nucleotide. I. Identification of intermediates. J Biol Chem 233: 488-492.

21. Preiss J, Handler P (1958) Biosynthesis of diphosphopyridine nucleotide. II. Enzymatic aspects. J Biol Chem 233: 493-500.

22. Sestini S, Ricci C, Micheli V, Pompucci G (1993) Nicotinamide mononucleotide adenylyltransferase activity in human erythrocytes. Arch Biochem Biophys 302: 206-211. doi:10.1006/abbi.1993.1200

23. Foster JW, Moat AG (1980) Nicotinamide adenine dinucleotide biosynthesis and pyridine nucleotide cycle metabolism in microbial systems. Microbiol Rev 44: 83-105.

24. Ginsburg H (2008) Malaria Parasite Metabolic Pathways. Available: http://sites. huji.ac.il/malaria/.

25. Plata G, Hsiao TL, Olszewski KL, Llinas M, Vitkup D (2010) Reconstruction and flux-balance analysis of the Plasmodium falciparum metabolic network. Mol Syst Biol 6: 408. doi:10.1038/msb.2010.60.

26. Sorci L, Pan Y, Eyobo Y, Rodionova I, Huang N, et al. (2009) Targeting NAD biosynthesis in bacterial pathogens: Structure-based development of inhibitors of nicotinate mononucleotide adenylyltransferase NadD. Chem Biol 16: 849-861. doi:10.1016/j.chembiol.2009.07.006.

27. Lau C, Niere M, Ziegler M (2009) The NMN/NaMN adenylyltransferase (NMNAT) protein family. Front Biosci 14: 410-431.

28. Lu S, Smith CD, Yang Z, Pruett PS, Nagy L, et al. (2008) Structure of nicotinic acid mononucleotide adenylyltransferase from Bacillus anthracis. Acta Crystallogr Sect F Struct Biol Cryst Commun 64: 893-898. doi:10.1107/ S1744309108029102.

29. D'Angelo I, Raffaelli N, Dabusti V, Lorenzi T, Magni G, et al. (2000) Structure of nicotinamide mononucleotide adenylyltransferase: a key enzyme in $\mathrm{NAD}(+)$ biosynthesis. Structure 8: 993-1004.

30. Zhang H, Zhou T, Kurnasov O, Cheek S, Grishin NV, et al. (2002) Crystal structures of $\mathrm{E}$. coli nicotinate mononucleotide adenylyltransferase and its complex with deamido-NAD. Structure 10: 69-79.
31. Larkin MA, Blackshields G, Brown NP, Chenna R, McGettigan PA, et al. (2007) Clustal W and Clustal X version 2.0. Bioinformatics 23: 2947-2948. doi:10.1093/bioinformatics/btm404.

32. Stancek M, Schnell R, Ryden-Aulin M (2005) Analysis of Escherichia coli nicotinate mononucleotide adenylyltransferase mutants in vivo and in vitro. BMC Biochem 6: 16. doi:10.1186/1471-2091-6-16.

33. Guzman LM, Belin D, Carson MJ, Beckwith J (1995) Tight regulation, modulation, and high-level expression by vectors containing the arabinose PBAD promoter. J Bacteriol 177: 4121-4130.

34. Huang N, Kolhatkar R, Eyobo Y, Sorci L, Rodionova I, et al. (2010) Complexes of bacterial nicotinate mononucleotide adenylyltransferase with inhibitors: implication for structure-based drug design and improvement. J Med Chem 53: 5229-5239. doi:10.1021/jm100377f.

35. Teng R, Junankar PR, Bubb WA, Rae C, Mercier P, et al. (2009) Metabolite profiling of the intraerythrocytic malaria parasite Plasmodium falciparum by (1)H NMR spectroscopy. NMR Biomed 22: 292-302. doi:10.1002/nbm.1323.

36. Jortzik E, Becker K (2012) Thioredoxin and glutathione systems in Plasmodium falciparum. Int J Med Microbiol 302: 187-194. doi:10.1016/ j.ijmm.2012.07.007.

37. Mazumdar J, Striepen B (2007) Make it or take it: fatty acid metabolism of apicomplexan parasites. Eukaryotic Cell 6: 1727-1735. doi:10.1128/EC.0025507

38. Yeh E, DeRisi JL (2011) Chemical rescue of malaria parasites lacking an apicoplast defines organelle function in blood-stage Plasmodium falciparum. PLoS Biol 9: e1001138. doi:10.1371/journal.pbio.1001138.

39. Bozdech Z, Llinas M, Pulliam BL, Wong ED, Zhu J, et al. (2003) The transcriptome of the intraerythrocytic developmental cycle of Plasmodium falciparum. PLoS Biol 1: E5. doi:10.1371/journal.pbio.0000005.

40. Llinas M, Bozdech Z, Wong ED, Adai AT, DeRisi JL (2006) Comparative whole genome transcriptome analysis of three Plasmodium falciparum strains. Nucleic Acids Res 34: 1166-1173. doi:10.1093/nar/gkj517.

41. Bozdech Z, Ginsburg H (2005) Data mining of the transcriptome of Plasmodium falciparum: the pentose phosphate pathway and ancillary processes. Malaria Journal 4: 17. doi:10.1186/1475-2875-4-17.

42. Divo AA, Geary TG, Davis NL, Jensen JB (1985) Nutritional requirements of Plasmodium falciparum in culture. I. Exogenously supplied dialyzable components necessary for continuous growth. J Protozool 32: 59-64.

43. Klein JR PW (1942) Determination of nicotinic acid in blood cells and plasma. Journal of Biological Chemistry 140: 423.

44. Gazanion E, Garcia D, Silvestre R, Gerard C, Guichou JF, et al. (2011) The Leishmania nicotinamidase is essential for NAD+ production and parasite proliferation. Mol Microbiol 82: 21-38. doi:10.1111/j.1365-2958.2011.07799.x.

45. Gazanion E, Seblova V, Votypka J, Vergnes B, Garcia D, et al. (2012) Leishmania infantum nicotinamidase is required for late-stage development in its natural sand fly vector, Phlebotomus perniciosus. Int J Parasitol 42: 323-327.

46. Belenky PA, Moga TG, Brenner C (2008) Saccharomyces cerevisiae YOR071C encodes the high affinity nicotinamide riboside transporter Nrt1. J Biol Chem 283: 8075-8079. doi:10.1074/jbc.C800021200.

47. Ma B, Pan SJ, Domergue R, Rigby T, Whiteway M, et al. (2009) High-affinity transporters for NAD+ precursors in Candida glabrata are regulated by Hst 1 and induced in response to niacin limitation. Mol Cell Biol 29: 4067-4079. doi:10.1128/MCB.01461-08

48. Jayaram HN, Kusumanchi P, Yalowitz JA (2011) NMNAT expression and its relation to NAD metabolism. Curr Med Chem 18: 1962-1972.

49. Petrelli R, Felczak K, Cappellacci L (2011) NMN/NaMN adenylyltransferase (NMNAT) and NAD kinase (NADK) inhibitors: chemistry and potential therapeutic applications. Curr Med Chem 18: 1973-1992.

50. Silvestrini F, Lasonder E, Olivieri A, Camarda G, van Schaijk B, et al. (2010) Protein export marks the early phase of gametocytogenesis of the human malaria parasite Plasmodium falciparum. Mol Cell Proteomics 9: 1437-1448. doi:10.1074/mcp.M900479-MCP200.

51. Treeck M, Sanders JL, Elias JE, Boothroyd JC (2011) The phosphoproteomes of Plasmodium falciparum and Toxoplasma gondii reveal unusual adaptations within and beyond the parasites' boundaries. Cell Host Microbe 10: 410-419. doi:10.1016/j.chom.2011.09.004.

52. Brayton KA, Lau AO, Herndon DR, Hannick L, Kappmeyer LS, et al. (2007) Genome sequence of Babesia bovis and comparative analysis of apicomplexan hemoprotozoa. PLoS Pathog 3: 1401-1413. doi:10.1371/journal.ppat.0030148.

53. Pain A, Renauld H, Berriman M, Murphy L, Yeats CA, et al. (2005) Genome of the host-cell transforming parasite Theileria annulata compared with T. parva. Science 309: 131-133. doi:10.1126/science.1110418.

54. Lambros C, Vanderberg JP (1979) Synchronization of Plasmodium falciparum erythrocytic stages in culture. J Parasitol 65: 418-420.

55. Trager W, Jensen JB (1976) Human malaria parasites in continuous culture. Science 193: 673-675.

56. Moore GE, Gerner RE, Franklin HA (1967) Culture of normal human leukocytes. JAMA 199: 519-524.

57. Fidock DA, Nomura T, Talley AK, Cooper RA, Dzekunov SM, et al. (2000) Mutations in the P. falciparum digestive vacuole transmembrane protein PfCRT and evidence for their role in chloroquine resistance. Mol Cell 6: 861-871.

58. Fidock DA, Wellems TE (1997) Transformation with human dihydrofolate reductase renders malaria parasites insensitive to WR99210 but does not affect the intrinsic activity of proguanil. Proc Natl Acad Sci U S A 94: 10931-10936. 
59. Saliba KJ, Horner HA, Kirk K (1998) Transport and metabolism of the essential vitamin pantothenic acid in human erythrocytes infected with the malaria parasite Plasmodium falciparum. J Biol Chem 273: 10190-10195.

60. Yu D, Ellis HM, Lee EC, Jenkins NA, Copeland NG, et al. (2000) An efficient recombination system for chromosome engineering in Escherichia coli. Proc Natl Acad Sci U S A 97: 5978-5983. doi:10.1073/pnas.100127597.
61. Datsenko KA, Wanner BL (2000) One-step inactivation of chromosomal genes in Escherichia coli K-12 using PCR products. Proc Natl Acad Sci U S A 97: 6640-6645. doi:10.1073/pnas.120163297.

62. Miller JH (1972) Experiments in Molecular Genetics. Cold Spring Harbor, NY: Cold Spring Harbor Laboratory.

63. Smilkstein M, Sriwilaijaroen N, Kelly JX, Wilairat P, Riscoe M (2004) Simple and inexpensive fluorescence-based technique for high-throughput antimalarial drug screening. Antimicrob Agents Chemother 48: 1803-1806. 